\title{
Part 12
}

\section{Meeting Summary}




\title{
IAU Colloquium 193 - A personal view
}

\author{
Steven D. Kawaler \\ Department of Physics and Astronomy, Iowa State University, Ames, \\ IA 50011, USA
}

\section{Introduction}

One of the more famous (or infamous) films of all time was Akira Kurosawa's Rashomon. In this film, Kurosawa tells the story of a terrible crime... and tells it four times. Each telling is from the perspective of a different character. In this masterful film the viewer is never quite sure what really happened; each of the protagonists tells the same tale but with their own personal interpretation.

Summarizing a week spent in the cold clear air of wintertime Christchurch (and that was inside the lecture hall) is indeed a daunting task. Each of us who was fortunate enough to have attended IAU Colloquium 193 came away from the meeting with our own impressions, highlights, and revelations. So by writing now about my own reflections, my goal is to rekindle yours, rather than persuade you that my summary is any more authoritative than one you might write. Of course, those of you reading this who were not at the conference are stuck with this summary - but by reading the preceding papers in this volume you, too, can have a sense of the variety we enjoyed.

Below, I start with a survey of some broad themes that emerged. A few results were of the "Gee Whiz" variety, and are outlined in the next section. A few old problems were revisited by several participants, and also some new problems have emerged, and I outline them next. After a nod to two very special participants in this Colloquium, I conclude with some final thoughts.

\section{Running themes}

Given the charge to this Colloquium by the organizers, many participants came from areas beyond the traditional crew of stellar pulsation researchers. We were, after all, given the task of addressing variable stars in the context of the Local Group of galaxies. As a result, we discussed topics in star formation, stellar populations, and galaxy evolution - all of which can benefit from creative application of knowledge of the properties of the host galaxy's variable star content.

On the first day, Antonio Aparicio gave a very illuminating overview of many issues facing the study of galaxy formation and the evolution of stellar populations that pulsating stars can help resolve. For example, the suite of dwarf spheroidal galaxies in our Local Group provides examples of the possible building blocks of more ordinary galaxies within the cold dark matter scenario, as described by Matteo Monelli and Márcio Catelan. Thus, they should contain only ancient stars from an early generation, as star formation long ago ceased. 
By identifying RR Lyrae and other variable stars, we can test that assumption. These two talks, and others, pointed out that the issue of the star formation history of dwarf spheroidal galaxies is not that simple, given the distribution of, for example, the RR Lyrae Oosterhoff types.

AGB stars can be carbon-rich or oxygen-rich, depending on their evolutionary stage and metallicity. With a diverse zoo of pulsating AGB stars at high luminosity, the AGB pulsators provide a probe of the metallicity and history of more distant galaxies. A number of contributions and talks explored ways to exploit the AGB variables for studies of galaxy evolution and chemical enrichment.

Beyond the broad focus of this Colloquium, we did enjoy a significant collection of stellar pulsation results of more general interest. With identification of nonradial pulsation in several new classes of stars, the key issue of pulsation mode identification appeared as a central issue in many talks. Long-period variable stars in the LMC show multiperiodic behavior occasionally, generating mode identification questions, but even the single-periodicity stars show conflicting signs. Similarly, the slowly pulsating B (SPB) stars, $\delta$ Scuti stars, $\beta$ Cephei stars, and EC 14026 stars require better mode identifications before full assaults by model matching can be used to probe their structures.

Back in 1999, a previous meeting (in Budapest) in this series was organized around the impact of large-scale surveys on variable star results. At that meeting, the OGLE and MACHO surveys had recently uncovered a huge number of new variable stars in the LMC and towards the galactic bulge. Now, four years later, the impacts of such surveys are really being felt. Many papers at our Colloquium detailed sophisticated analyses of the data from large surveys, and have provided brand-new insights into stellar pulsation. It is a different ball game now, with statistics from several thousands of members of a given class of pulsating star, rather than the dozens or even hundreds of a decade ago. Such surveys have also revealed that the long-period variable population includes not only AGB stars but also a significant number of first-ascent RGB variables. Laszlo Kiss and others showed that the sequences of RGB variables allow us to have a new probe of globular clusters, for example.

And, of course, you can also remember other themes . . .

\section{Gee-whiz results}

In the very first lecture of this colloquium, Joergen Christensen-Dalsgaard helped fire us up with an overview of current theoretical developments in variable star research. In the process, he previewed a number of later talks that presented some new observational results. Many of them were quite breathtaking - and here again, the Rashomon effect comes into play. I'll mention a few of those and some others that I found intriguing. Each of us went home wondering about the set of new results that most resonates with our own world view. Here's mine.

The influence of turbulent driving of nonradial modes is evident in the Sun, and (as found recently) other solar-type stars. But the fact that the same effect can work in more evolved stars, such as core helium burning stars and even AGB stars, is mounting. Before this year, when one saw a collection of a few Fourier 
transforms of stars in a single figure, it represented a class of variable stars with similar properties. Such diagrams are frequently shown in review talks on white dwarf stars, $\delta$ Scuti stars, or pulsating sdB stars - classes with members that are multiperiodic, and have uniform-quality data. What was new and exciting this time was Joergen's display of a figure (originally drafted by Tim Bedding) of 5 power spectra of solar-type oscillations in other stars! In the time between the Leuven meeting and this one, the progress in measuring solar-like oscillations has been astounding. In the same talk, Joergen showed a radial velocity curve for $\alpha \mathrm{Cen} \mathrm{A}$ - one where the signal-to-noise was sufficiently high that you could see the wiggles - at the $\mathrm{cm} \mathrm{s}^{-1}$ level. Similar-looking oscillations have been seen in the helium core burning star $\chi$ Hydri, and perhaps in SR variables.

Nonradial pulsations seem to be present in stars long thought to be simple radial pulsators. Tomasz Mizerski showed evidence of multiperiodic pulsation in RR Lyrae stars that exhibit the Blazhko effect. This is not an isolated star or two; hundreds of such stars have been found in the galactic bulge. Pawel Moskalik used OGLEII data on the LMC to show that many Cepheids show multiple periodicities that cannot be explained by radial pulsation alone. In both cases, these "classical" variable stars show rotational splitting characteristic of nonradial pulsations.

I teach a large introductory astronomy course at my university, and always introduce the stellar astronomy section with a statement that: aside from our Sun, we have not seen the surface of any other star. I'll need to modify that statement now, based on the results of milli-arcsecond maser measurements of AGB stars that Harm Habing reviewed. I found it truly amazing that using radio observations, the structure of the masing region could be determined, yet the position of the star itself was more uncertain! Additional results he showed included limits on the magnetic field of AGB stars at the milli-Gauss level. In addition, Nicolas Nardetto showed that interferometric measurements of Cepheid radii are now being made, giving a fundamental connection between the P-L diagram and theory through the period-radius relation.

Denis Sullivan reviewed work that he and the University of Texas group are doing that may lead to the detection of Earth-like planets around white dwarf stars. Some pulsating white dwarfs have stable-enough pulsations (in amplitude, period, and phase) that the pulsations serve as a precision clock. In a way analogous to pulsar studies, the white dwarf pulsations can reveal reflex orbital motion of the star as planets orbit it. Recent discoveries of a number of such stable pulsators by the Sloan Digital Sky Survey imply that reasonable statistics on the incidence of planets may be forthcoming.

As useful as Cepheids have been as a cosmological distance probe, they are relatively rare stars. Using large-scale survey data over the past decade, work by Peter Wood and others has revealed that Mira variables show a valid periodluminosity relation, and therefore provide another long-range distance indicator. How long-range? Mike Feast showed a terrific example of the possibilities of this technique with a Mira P-L distance to Centaurus A.

Peter Wood has investigated the mystery of long secondary periods in Mira variables. Again, the availability of the MACHO data on Miras in the LMC gives us a window on these stars - and shows that many of them display periods that are many times longer than the radial fundamental period. He considered 
several possible explanations, none of which is completely satisfactory - so the mystery remains. (Full disclosure: I've collaborated with Peter on one aspect of this problem.)

Another surprise came from Nancy Evans as part of her discussion of the masses of Cepheid variables. In addition to her results on S Mus, she pointed out that an unusually large percentage of Cepheids appear to be in triple-star systems. Another talk, by Brian Warner, also involved pulsating stars in binary systems. He has been finding an increasing number of cataclysmic variables that contain a white dwarf undergoing nonradial pulsation.

And of course you remember many other gee-whiz results . . .

\section{Somethings old}

For nearly 100 years, the period-luminosity relation for Cepheid variables has been a key aspect of stellar and extragalactic astrophysics. It continues to be refined and explored, and some new results on this old problem were presented here. For example, we can obtain a thorough census of Cepheids within galaxies in the local group, where distances are known by other means. Resolving them from nearby stars in their host galaxies allows us to make new tests of observational selection effects that play some role in their use in more distant systems, as discussed by a number of papers here. The dependence of the Cepheid P-L-C relation on metallicity can also be examined, and reports from several investigators here explored the impact of metallicity on Cepheid distances.

Lucas Macri provided an interesting historical note about on Hubble's identification of variable star V19 in M33 - Hubble identified it as a 55-d Cepheid, but Macri and collaborators saw no variation. Allan Sandage is following up on this mystery by reanalyzing Hubble's original photographic plates. Now that is a bit of history!

Ed Guinan reminded us of the importance of eclipsing binary stars to the development of stellar evolution theory - they provide a critical suite of physical parameters for the stars involved. Now, this same technique can be used, in some sense, in reverse, to provide a high-precision distance to the LMC. The value of such a high-precision distance, obtained using "old fashioned celestial mechanics" is that it reduces the largest single component of the current uncertainty to the extragalactic distance scale: the uncertainty in the distance to the LMC.

Joyce Guzik outlined her attempts to match the observed pulsation periods of $\delta$ Scuti stars with models. She illustrated a problem that we have had with such stars for decades - matching the observed periods with precision approaching the observational uncertainty remains beyond our capabilities. This is a problem that is similar to the early days of helioseismology - but with many fewer modes we should be able to do better. The fact that we cannot is telling us something fundamental about stars. In a similar vein, the mystery of the origin of blue loops during helium core burning remains, and was discussed here by Santi Cassisi and Joyce Guzik. First identified in the early days of computational stellar evolution, the physics behind the blue loops, and their sensitivity 
to the details of the input physics and computational methods, confounds our abilities to match the $\mathrm{H}-\mathrm{R}$ diagram positions of evolving stars.

And other old "friends" of yours were actively discussed . . .

\section{Somethings new}

Along with some new twists on some old problems as described above, we saw the introduction, or at least maturation, of some new problems and puzzles for active investigation. I already mentioned some of these in previous sections, such as the spectacular progress in seeing and now studying solar-like oscillations on solar-type (and other kinds of) stars, the use of RGB variables as distance indicators for globular clusters, and of Miras for extragalactic distances. Here are some others.

Conny Aerts and Gerald Handler, in a masterful team presentation, described the observations, analysis, and modeling of many nonradial modes in the massive star HD blah-blah-blah (HD 129929; V836 Cen). Their analysis suggests that the star is undergoing differential rotation with depth, and represents (perhaps) the birth of massive star asteroseismology.

Not too long ago, the idea of a P-L relation for Mira variables seemed vague or fuzzy, but following the MACHO and OGLE surveys, the wealth of data on LPVs revealed families of $\mathrm{P}-\mathrm{L}$ relations. The principal tool for classifying these is now known as a "Peter Wood diagram" and was employed by many presenters at this Colloquium (including Peter Wood). This diagram turns out to be a key to identifying the evolutionary state of LPVs in distant stellar populations, and so on. Studies in this realm have established its universality (and thus justifying the use of the Mira P-L relation for extragalactic distances), yet the explanation for the various groups seen in the diagram remains somewhat speculative.

We heard from several people of the need for including diffusion in evolution and pulsation calculations. While this is not really new, we are now, finally, at a point where the effects of diffusion cannot be ignored - not just in trying to understand the details, but in trying to understand fundamental properties such as excitation mechanisms.

Likewise, the roAp stars show peculiar surface abundances and strong magnetic fields. Yet, despite these complications, progress continues on studying the connection between magnetic fields and pulsation (as described by Lionel Bigot and Hideyuki Saio). Another new mystery has emerged from these stars. Mikhail Sachkov showed results of time-resolved radial velocity studies of the metal lines in roAp stars. We all wonder at the fact that large variations were seen in some of the rare Earth lines, yet small (if any) variations were seen in others. Hiromoto Shibahashi (at least) is trying to do something about it.

And, in particular, you recall other new puzzles for us to ponder . . . 


\section{Heroes among us}

The ongoing series of "Los Alamos Pulsation Meetings" that began in the second half of the last century, and continues today, shows that the study of variable stars has been, and continues to be, one of the foundations of astrophysics. From the very start of my research career, I have been in awe of the work by the founders of modern stellar astrophysics. As my focus drew to stellar pulsation, that feeling of awe has been reinforced again and again. Here in Christchurch, we had among us two people who have been important parts of the story of variable stars for decades.

My own entry into the field was the result of a fortunate accident. Back before I attended college, my interest in astronomy was as an amateur observer. Research, to me, was finding and tracking asteroids using a small telescope in my backyard, with the occasional lunar occultation to spice things up. A very early influence came from Joe Patterson who, before he even started in his own research career, ran a summer camp for astronomy "nerds" that I attended for two summers as a teen. Some of my colleagues in astronomy today are also campers influenced by Joe.

When I went to college, I worked with the terrific group of planetary scientists assembled at Cornell, and with Frank Drake doing radio astronomy. Graduate school was the obvious next step, so I ended up at the University of Texas where both fields were (I thought) widely represented.

My love of observing beneath the stars remains, despite a career which has taken me in the direction of theory. Upon arrival at Texas, I was captivated by the charismatic lectures by Craig Wheeler in the introductory graduate course in stellar structure and evolution. I soon got hooked on stellar evolution theory, and then Don Winget arrived at Texas. All the pieces were in place, and my thesis work on pulsating white dwarf stars with Don (and many other colleagues) set the stage for my professional career ever since (though I do occasionally sneak out to my back yard with my small telescope and find an asteroid or two to track).

So, though I started as a "typical" amateur astronomer, I ended up working in the area of computational stellar astrophysics. At the Christchurch meeting are two people who embody excellence in both of those areas. They come to this meeting and this subject from very different directions, and their contributions are in very different arenas, but individually they represent the fact that our field relies on observers and theorists. The balance is nearly perfect. At the risk of further embarrassing both of these very modest gentlemen, I want to single out Norman Baker and Albert Jones.

Norman Baker's contributions include his early studies of Cepheids - including early successful nonlinear hydrodynamic computations of Cepheid light and velocity curves at a time when computers were room-size but had the memory and power of modern digital watches. The results he obtained in those pioneering days remain valid today, and constitute the fundamental benchmark by which our understanding of other pulsating stars is measured. The numerical techniques developed by Norman Baker included a generalized Newton-Rapheson integrator for relaxation solutions to differential equations that forms the core of many of our modern pulsation and evolution codes. Yet besides his technical 
wizardry, Norman Baker's focus on the science of these stars has been incisive since the 1960s.

When most people think of what an astronomer is like, they probably imagine someone spending long hours at the eyepiece of a telescope, measuring something-or-other about stars. In a real sense, they are picturing what Albert Jones has faithfully been doing for over 50 years. Albert's career as an astronomer has provided a treasure of observations that graces the databases of international variable star associations. His data are essential to researchers who study a variety of variable star types. And he has done this while leading an otherwise normal life, with a normal job during the day! Working with data obtained by Albert on semi-regular variable stars such as L2 Puppis, I have seen firsthand the high quality and uniformity of the observations he has made - and there are countless other research astronomers who have taken advantage of the remarkable data that he has produced during his long career. His presence in Christchurch gave many of us the chance to meet him (some of us for the first time), and gave us all a chance to thank him for his tireless efforts.

\section{Conclusion}

Indeed; this was quite a week. While Kurosawa's Rashomon illustrated the ambiguities of a terrible event using but four participants, IAU 193 was a very pleasant and engaging conference with well over 100 participants. But unlike in Rashomon, I think it is safe to say that all of us came away from this conference in various degrees of elation, refreshed by the vitality of the field of stellar pulsation.

Despite over 40 years of time passing since the detailed exposure of the inner workings of Cepheid variables, they and other classes of variable stars remain mysterious and fascinating. Using variable stars that we could only study, in years past, in the solar neighborhood, we can probe the histories of galaxies in the Local Group. What is next? 


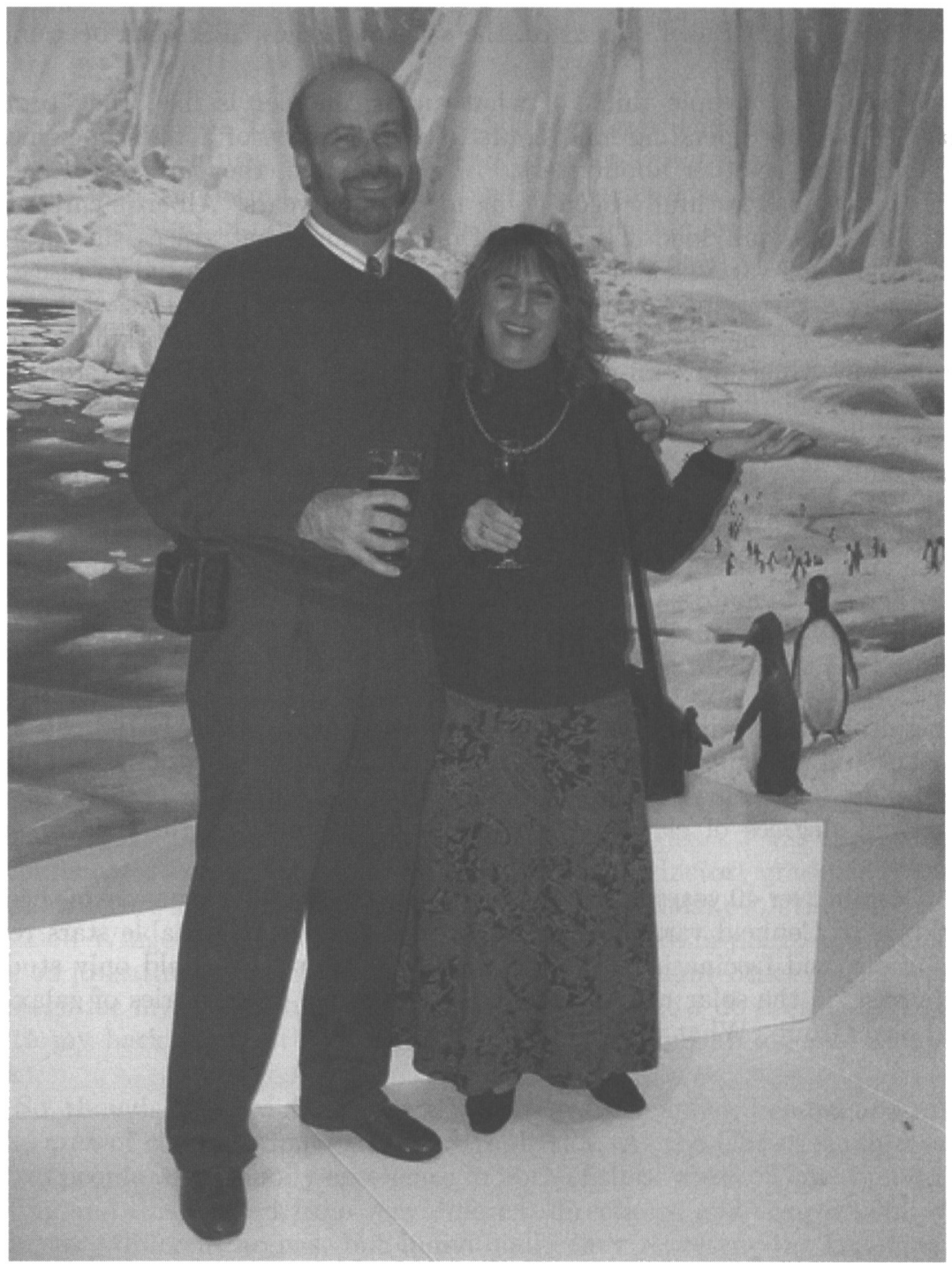

Steve and Leslie Kawaler 


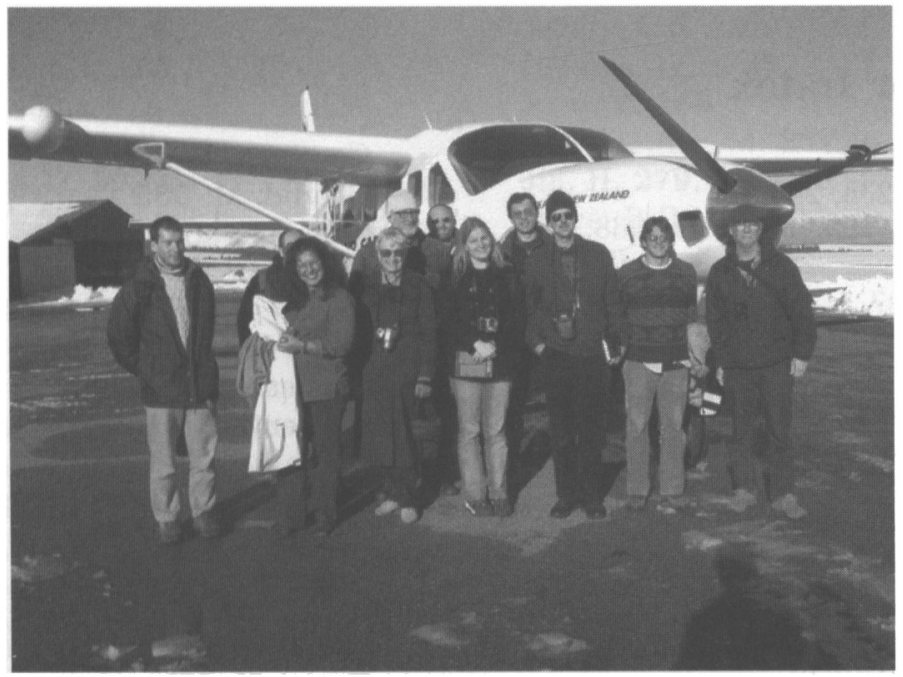

Scenic flight over Lake Tekapo, Mt Cook and the Southern Alps

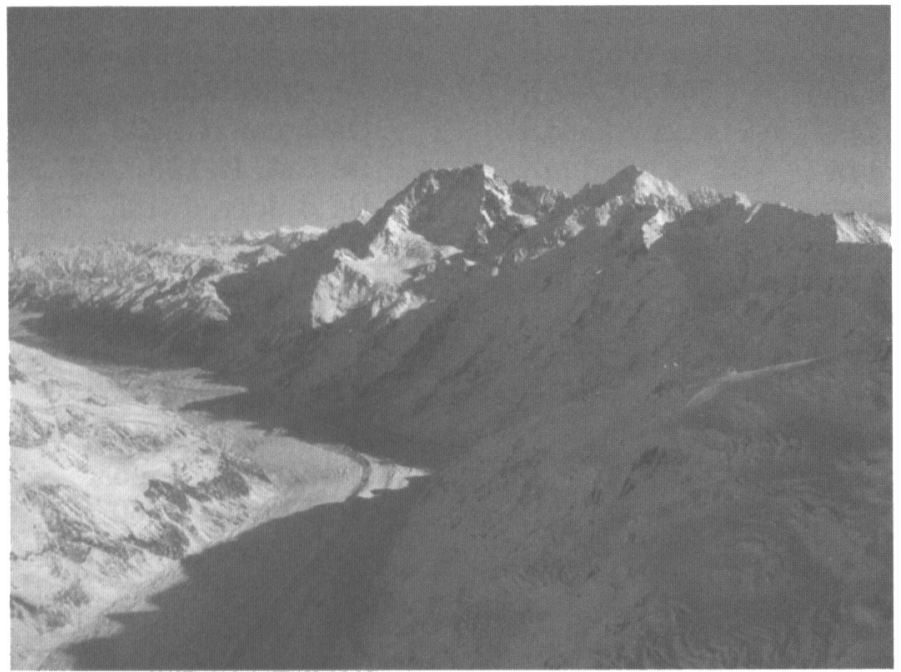

A view from the flight on a perfect photometric day 